\title{
Biomimetic sensing based on chemically induced assembly of a signaling DNA aptamer on a fluid bilayer membrane
}

\author{
Neeshma Dave and Juewen Liu*a \\ Received $4^{\text {th }}$ January 2012, Accepted $17^{\text {th }}$ February 2012 \\ ${ }_{5}$ DOI: 0.1039/c2cc00070a
}

The adenosine aptamer was split into two halves and linked to a fluid liposome surface; addition of adenosine resulted in aptamer assembly, which did not occur if the split aptamer was attached to silica nanoparticles, demonstrating the ${ }_{10}$ feasibility of using aptamer probes to study diffusion within lipid membranes.

The cell membrane is composed of a lipid bilayer with associated proteins. In addition to serving as a barrier to encase the cellular content, the fluid bilayer allows membrane proteins to reorganize and assemble in response to chemical stimuli for complex functions such as cellular signaling and endocytosis. ${ }^{1}$ Using such a dynamic mechanism, numerous proteins and small molecules can be detected by the cell. The detection is also reversible and assembled receptors can disassemble at reduced stimulus concentration. It has also become clear in the past two decades that lipid composition is also important for the function of membrane proteins. For example, lipid raft formation and phase separation are suggested to be crucial for cell signaling. ${ }^{2,3}$ Due to the complexity of the membrane protein system, studying their assembly remains difficult. ${ }^{4}$

To gain insight into membrane biophysics, ligand reorganization within lipid bilayers has been studied in several model systems including metal chelating lipids and metal binding proteins ${ }^{1,5,6}$. For example, a lipid probe was prepared to contain a pyrene in the hydrophobic tail and a $\mathrm{Cu}$ (II)-iminodiacetate in the head group. In the presence of a poly-histidine peptide, this lipid aggregated to form pyrene excimers. While valuable insights have been gained, it is desirable to expand the range of stimuli to other compounds such as small molecule metabolites.

Aptamers are nucleic acid based binding molecules that can be selected to bind to essentially any molecule of choice. ${ }^{7}$ Many aptamers have been made into biosensors. ${ }^{8}$ DNA conjugation to liposome has also been well established. ${ }^{9}$ Taking advantage of these developments, we herein report the attachment of an engineered adenosine aptamer on a liposome. The original aptamer was split into two halves; these two halves can assemble into the full aptamer in the 45 presence of adenosine on lipid bilayer. However, no assembly was detected when the aptamer was immobilized on a silica nanoparticle, where surface diffusion was eliminated. This work has established the feasibility of using aptamers for studying lateral diffusion in membrane. Based on this specific $5_{0}$ binding interaction, this split aptamer system has been tested as a biosensor for adenosine detection.
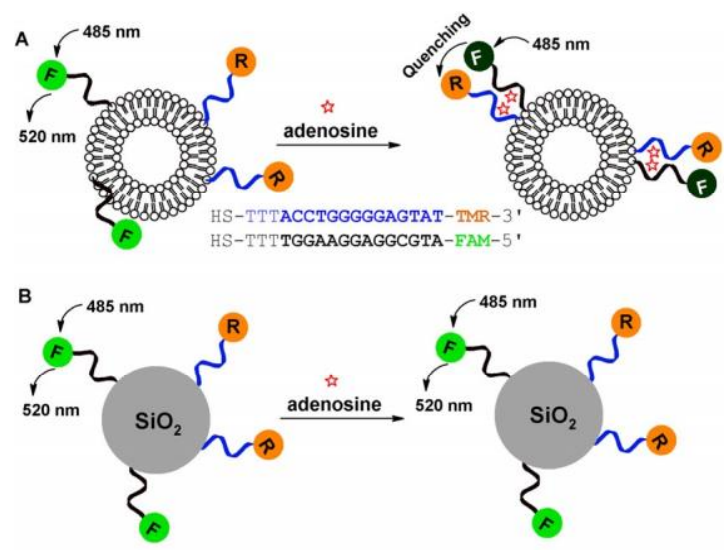

Figure 1. (A) Schematic presentation of adenosine induced assembly of split fluorescent aptamers on liposome surface. 55 (B) If immobilized on silica nanoparticles, the aptamers cannot diffuse or assemble in the presence of adenosine.

Figure 1A shows the sensor design. The liposome contained 95\% 1,2-dioleoyl-sn-glycero-3-phosphocholine (DOPC) with 5\% 1,2-dioleoyl-sn-glycero-3-phosphoethanolamine-N-[4(pmaleimidophenyl)butyramide] (MPB-PE). DOPC has a phase transition temperature of $-20{ }^{\circ} \mathrm{C}$ and therefore is fluid at room temperature. The MPB group allows covalently attachment of thiolmodified DNA. The liposome was prepared using the standard extrusion method through a $100 \mathrm{~nm}$ pore size membrane and the average liposome size was determined to be $142 \mathrm{~nm}$ using dynamic light scattering (Figure S1, ESI). In this study, we chose to use the adenosine/ATP aptamer because it has been widely used as a model for designing biosensors. In addition, splitting the aptamer into two halves has also been demonstrated. ${ }^{11-14}$ We labeled each split aptamer with a thiol group and a fluorophore. A 6carboxyfluorescein (FAM) was labeled on the 5 '-end of one DNA as the FRET donor; a carboxytetramethylrhodamine (TMR) was labeled on the $3^{\prime}$-end of the other half to serve as the acceptor. After incubating the DNAs with liposome overnight, non-reacted free DNAs were removed by ultracentrifugation. 
The aptamerfunctionalized liposomes were then re-dispersed in buffer A (20 mM NaCl, $10 \mathrm{mM}$ HEPES, pH 7.6) for further studies. These two DNAs were sparsely attached on the DOPC liposome with a density of $\sim 60$ FAM-labeled DNA and $~ 120$ TMR-labeled DNA per liposome (Figure S2, ESI). We used higher TMR-DNA to achieve more efficient energy transfer (Figure S3, ESI). Considering the liposome diameter to be $\sim 140 \mathrm{~nm}$ and the surface area to be $61544 \mathrm{~nm}^{2}$, each DNA occupies an area of $\sim 341 \mathrm{~nm}^{2}$. Therefore, the distance between each DNA is $\sim 18.5 \mathrm{~nm}$ if the DNA molecules are 10 evenly distributed without any interaction.
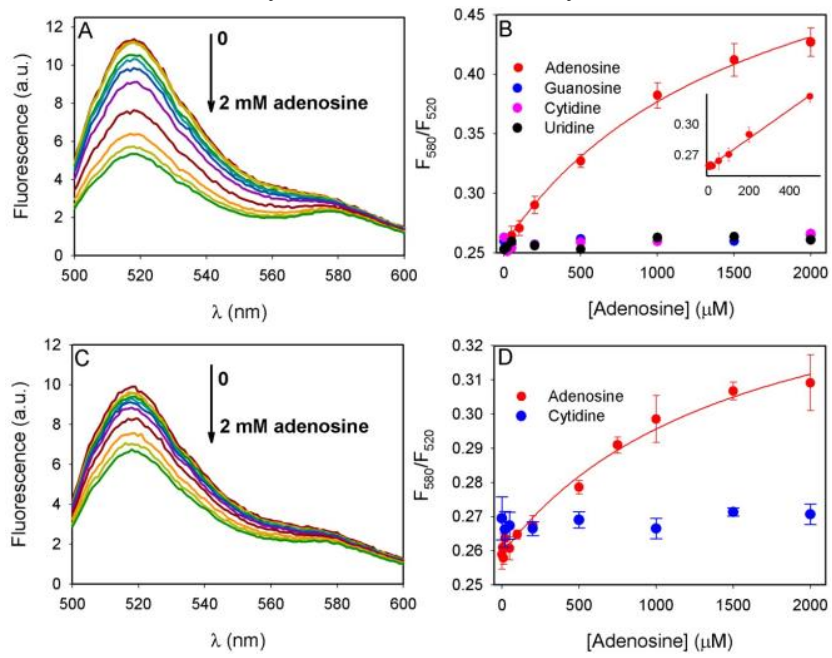

Figure 2. Fluorescence spectra for DOPC (A) and DPPC (C) and fluorescence ratio for DOPC (B) and DPPC (D) of the split aptamer functionalized liposome titration with adenosine and other nucleosides. Inset of (B): the low adenosine region.

Since the Förster distance $\left(R_{0}\right)$ for the FAM/TMR pair is $\sim 5.5 \mathrm{~nm}$, at a separation of $\sim 18.5 \mathrm{~nm}(\sim 3 R 0)$, the FRET efficiency between the two fluorophores should be very low. 20 Upon exciting at 490 $\mathrm{nm}$, a strong peak at $520 \mathrm{~nm}$ due to FAM emission and a small shoulder at $580 \mathrm{~nm}$ from TMR were observed (Figure 2A). We measured the fluorescence ratio of $580 \mathrm{~nm}$ over $520 \mathrm{~nm}$ to be 0.25 (Figure 2B). This ratio was only slightly higher than that for the free non 25 immobilized DNAs dissolved in solution (ratio $=\sim 0.22$, Figure 3B), indicating that there was indeed very little energy transfer and the DNAs were well separated. Addition of adenosine resulted in a gradual decrease in the $520 \mathrm{~nm}$ peak while the 580 nm peak changed very little (Figure $2 \mathrm{~A}$ ), ${ }_{30}$ suggesting that the quenching of the FAM should be due to ground state complex formation or static quenching. This was likely to occur because after binding adenosine, the FAM and TMR dyes were right next to each other (Figure 1A). Fluorescence lifetime measurement also supported this static quenching mechanism (Figure S4, ESI). The fluorescence ratio of $580 \mathrm{~nm}$ over $520 \mathrm{~nm}$ gradually increased with increasing adenosine concentration (Figure 2B, red dots); the apparent dissociation constant $\left(K_{d}\right)$ was calculated to be $1.65 \mathrm{mM}$, which was significantly higher compared to the $\sim 10 \mu \mathrm{M}_{40}$ of the original non-split aptamer. This is likely to be related to the fact that the split aptamer forms a ternary reaction. From the titration curve, the detection limit was calculated to be $60 \mu \mathrm{M}$ adenosine, at which the signal was higher than three times the background variation (Figure 2B, inset). The signal 45 change was instantaneously as shown by the kinetic studies (Figure S5, ESI). As a control, we prepared liposomes functionalized only with the FAM-labeled DNA. Addition of adenosine caused only a slight decrease of its fluorescence due to dilution (Figure S6B, ESI). Therefore, the quenching 50 by adenosine in Figure 2A can only be attributed to aptamer assembly. If other ribonucleosides were added, there was little change in the ratio (Figure 2B), confirming that the split aptamers still had high specificity.

By confining ligands in the lipid bilayer, a high local concentration can be achieved even though the overall bulk solution concentration may be low. To test this feature, we reduced the liposome concentration four times and similar adenosine-dependent binding curve was still obtained (Figure S7, ESI). On the other hand, if the two free DNAs were dissolved in the same buffer, we observed no adenosinedependent fluorescence ratio change (Figure 3A, B), suggesting that the two DNAs were too dilute to bind. Sensors based on splitting the adenosine aptamer have been reported previously in several systems where high DNA concentrations, ${ }^{11}$ enlongated binding arms, ${ }^{12,13}$ or multivalent binding ${ }^{14}$ has to be used to achieve adenosine binding. In all of these previous examples, the bulk DNA concentration was crucial for binding. In our liposome system, the concentration of aptamer was required only for detection. In theory, even asingle liposome should also work the same way as long as it can be detected. On the other hand, if we keep the bulk DNA concentration the same but dilute the DNA density on the liposome five times, an adverse effect on binding was observed with $K_{d}=\sim 9 \mathrm{mM}$ (Figure S8, ESI). On a cell membrane, there are always multiple copies of the same receptors. In addition to achieving multivalent binding, ${ }^{15}$ another function might be to achieve a high enough concentration to facilitate binding and selfassembly.

To understand the effect of lipid fluidity, we also prepared 1,2-dipalmitoyl-sn-glycero-3-phosphocholine (DPPC) with 5\% MPB-PE. DPPC has a phase transition temperature of $41{ }^{\circ} \mathrm{C}$ and thus is in the gel phase at $25^{\circ} \mathrm{C}$. The attached split aptamers showed a similar $K_{d}$ of $1.55 \mathrm{mM}$ adenosine although the amount of quenching was lower compared to the case of DOPC. The diffusion coefficients for DOPC and DPPC differ by just one-fold and therefore DNA on DPPC was still quite mobile, ${ }^{16}$ which may explain the similar $K_{d}$ in both cases.

To completely eliminate lateral diffusion, we tested $100 \mathrm{~nm}$ silica nanoparticles for immobilization. The silica particles 90 contained amine groups on the surface and a bifunctional crosslinker was first reacted with the silica particles and then the purified particles were reacted with the thiolated DNAs. Each silica nanoparticle was estimated to contain $~ 50$ FAMlabeled DNA and 100 TMR DNA. Therefore the DNA 95 density was similar to that on the liposome. As can be observed from Figure 3C, D, the aptamer also failed to bind adenosine and little change in the FRET ratio was observed. This was attributed to the inability of the anchored DNAs to diffuse and assemble in response to adenosine because of the static nature of the silica surface, confirming that the fluidity of the bilayer membrane was extremely important for the molecular recognition to effectively take place. 

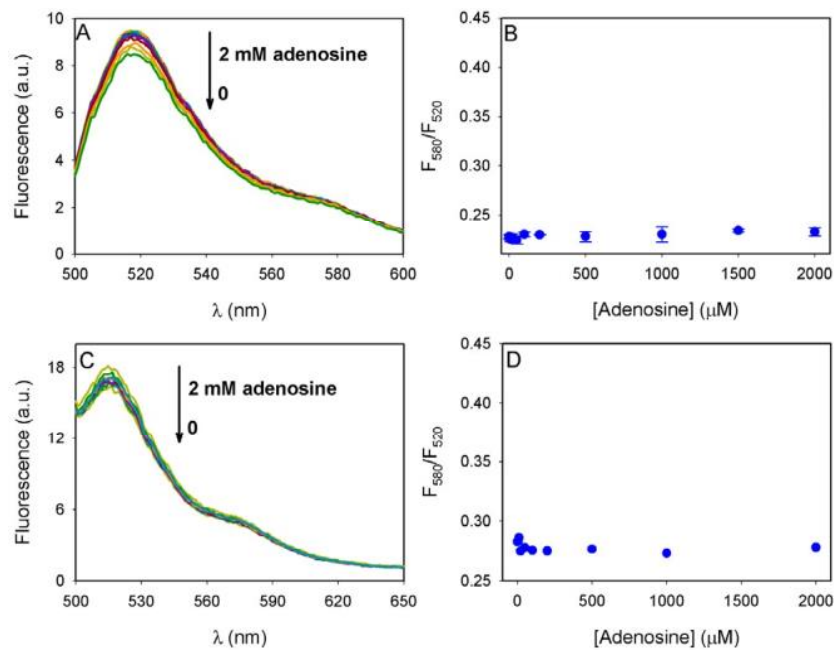

Figure 3. Fluorescence spectra (A) and fluorescence ratio (B) 5 of free split aptamers in solution titration with adenosine. Fluorescence spectra (C) and fluorescence ratio (D) of the split aptamers immobilized on silica nanoparticles titration with adenosine.

In summary, we have constructed a biomimetic sensing system taking advantage of the fluid nature of the lipid bilayer membrane. The cell membrane is known to encapsulate the cellular content to increase their effective concentration. This concept has been utilized by liposome-based materialssynthesis, drug delivery, and fusion studies. The membrane itself is also capable of achieving similar goals. Immobilization of DNA aptamers on the liposome surface allows the construction of an effective biosensor. At the same time, this system can also serve as a model to study variousbiophysical features of receptor reorganization and assembly in the cell membrane.

Funding for this work is from the University of Waterloo, the Canadian Foundation for Innovation, and the Discovery Grant of the Natural Sciences and Engineering Research Council (NSERC) of Canada. J. Liu receives Early Researcher Award from the Ontario Ministry of Research and Innovation.

\section{Notes and references}

Department of Chemistry, Waterloo Institute for Nanotechnology, University of Waterloo, Waterloo, Ontario, N2L 3G1, Canada. Fax: 519 7460435; Tel: 5198884567 Ext.38919; E-mail: liujw@uwaterloo.ca

1 D. W. Pack, K. Ng, K. M. Maloney and F. H. Arnold, Supramol. Sci., 1997, 4, 3.

2 K. Simons and D. Toomre, Nat. Rev. Mol. Cell Biol., 2000, 1, 31.

3 E. L. Elson, E. Fried, J. E. Dolbow and G. M. Genin, in Ann. Rev. Biophys., 2010, 39, 207.
4 X. Periole, T. Huber, S. J. Marrink and T. P. Sakmar, J. Am. Chem. Soc., 2007, 129, 10126.

5 K. M. Maloney, D. R. Shnek, D. Y. Sasaki and F. H. Arnold, Chem. Biol., 1996, 3, 185.

6 D. Rapaport and Y. Shai, J. Biol. Chem., 1992, 267, 6502.

7 D. S. Wilson and J. W. Szostak, Annu. Rev. Biochem., 1999, 68, 611.

8 (a) J. Liu, Z. Cao and Y. Lu, Chem. Rev., 2009, 109, 1948; (b) E. J. Cho, J.-W. Lee and A. D. Ellington, Annu. Rev. Anal. Chem., 2009, 2, 241; (c) D. Li, S. P. Song and C. H. Fan, Acc. Chem. Res., 2010, 43, 631; (d) N. K. Navani and Y. Li, Curr. Opin. Chem. Biol., 2006, 10, 272; (e) E. Katz and I. Willner, Angew. Chem., Int. Ed., 2004, 43, 6042; (f) A. A. Lubin and K. W. Plaxco, Acc. Chem. Res., 2010, 43, 496; (g) M. Famulok, J. S. Hartig and G. Mayer, Chem. Rev.,

2007, 107, 3715; (h) M. Zhou and S. Dong, Acc. Chem. Res., 2011, 44, 1232; (i) H. Wang, R. H. Yang, L. Yang and W. H. Tan, ACS Nano, 2009, 3, 2451.

9 (a) F. Patolsky, A. Lichtenstein and I. Willner, J. Am. Chem. Soc., 1999, 122, 418; (b) F. Patolsky, A. Lichtenstein and I. Willner, J.

Am. Chem. Soc., 2001, 123, 5194; (c) Y. H. M. Chan, B. van Lengerich and S. G. Boxer, Proc. Natl. Acad. Sci. U.S.A., 2009, 106, 979; (d) C. Yoshina-Ishii, G. P. Miller, M. L. Kraft, E. T. Kool and S. G. Boxer, J. Am. Chem. Soc., 2005, 127, 1356; (e) A. S. Achalkumar, R. J. Bushby and S. D. Evans, Soft Matter, 2010, 6, 6036; (f) G. Stengel, R. Zahn and F. Hook, J. Am. Chem. Soc., 2007, 129, 9584; (g) N. Dave and J. Liu, Acs Nano, 2011, 5, 1304; (h) N. Dave and J. Liu, Adv. Mater., 2011, 23, 3182; (i) P. A. Beales and T. K. Vanderlick, J. Phys. Chem. A, 2007, 111, 12372.

10 (a) S. D. Jhaveri, R. Kirby, R. Conrad, E. J. Maglott, M. Bowser, R. T. Kennedy, G. Glick and A. D. Ellington, J. Am. Chem. Soc., 2000, 122, 2469; (b) R. Nutiu and Y. Li, J. Am. Chem. Soc., 2003, 125, 4771; (c) J. Liu and Y. Lu, Angew. Chem., Int. Ed., 2006, 45, 90; (d) N. Lu, C. Y. Shao and Z. X. Deng, Chem. Comm., 2008, 6161; (e) S. J. Chen, Y. F. Huang, C. C. Huang, K. H. Lee, Z. H. Lin and H. T. Chang, Biosens. Bioelectron., 2008, 23, 1749; (f) J. Wang, L. H. 75 Wang, X. F. Liu, Z. Q. Liang, S. P. Song, W. X. Li, G. X. Li and C. H. Fan, Adv. Mater., 2007, 19, 3943; (g) Y. Xiang, A. J. Tong and Y. Lu, J. Am. Chem. Soc., 2009, 131, 15352.

11 (a) R. Freeman, Y. Li, R. Tel-Vered, E. Sharon, J. Elbaz and I. Willner, Analyst, 2009, 134, 653; (b) R. P. Fahlman and D. Sen, J. 80 Am. Chem. Soc., 2002, 124, 4610; (c) J. W. Liu and Y. Lu, Anal. Chem., 2004, 76, 1627; (d) X. Zuo, Y. Xiao and K. W. Plaxco, J. Am. Chem. Soc., 2009, 131, 6944; (e) Z. Lin, F. Luo, Q. Liu, L. Chen, B. Qiu, Z. Cai and G. Chen, Chem. Comm., 2011, 47. 12 M. N. Stojanovic, P. de Prada and D. W. Landry, J. Am. Chem. Soc., ${ }_{5} 2000$, 122, 11547.

13 Z. Y. Liu, W. Zhang, L. Z. Hu, H. J. Li, S. Y. Zhu and G. B. Xu, Chem. Eur. J., 2010, 16, 13356.

14 F. Li, J. Zhang, X. N. Cao, L. H. Wang, D. Li, S. P. Song, B. C. Ye and C. H. Fan, Analyst, 2009, 134, 1355.

15 M. Mammen, S. K. Choi and G. M. Whitesides, Angew. Chem. Int. Ed., 1998, 37, 2755.

16 A. Benda, M. Benes, V. Marecek, A. Lhotsky, W. T. Hermens and M. Hof, Langmuir, 2003, 19, 4120. 